\title{
SATISFAÇÃO DE UMA EQUIPE DE ENFERMAGEM QUANTO A PROFISSÃO E EMPREGO NUM HOSPITAL DO SUL DO ESTADO DO RIO GRANDE DO SUL
}

[Satisfaction os a team of nursing as the profession and job in a hospital of the state of Rio Grande do Sul]

\author{
Diana Cecagno* \\ Susana Cecagno** \\ Hedi Crecencia Hecler de Siqueira***
}

RESUMO: A insatisfação profissional pode ocasionar uma multiplicidade de fatores negativos interferindo na qualidade nos serviços. Faz-se necessário manter prazer no trabalho, pois a satisfação e a auto-realização serão maiores quanto maior o seu envolvimento. Este estudo objetivou investigar a satisfação profissional e empregatícia de trabalhadores de enfermagem num Hospital do Sul/RS. Caracterizou-se como quantitativo, utilizando-se um questionário fechado, com 50 profissionais de enfermagem. Verificou-se que as médias, em anos, das variáveis analisadas foram: 33,4 (idade), 6,2 (tempo de formado) e 5,8 (tempo de trabalho). A distribuição por função foi de $22 \%$ enfermeiros, $16 \%$ técnicos e $62 \%$ auxiliares. O valor médio atribuído quanto a satisfação profissional foram $7,8,8,3$ e 8,5 e para o vínculo empregatício 6,8, 7,9 e 8,3 para, enfermeiros, técnicos e auxiliares, respectivamente. Conclui-se que os técnicos e auxiliares apresentam maior grau de satisfação que o enfermeiro. Esse trabalho instiga-nos a investigar os motivos influenciadores da satisfação profissional e empregatícia da equipe de enfermagem.

PALAVRAS-CHAVE: satisfação, trabalho, enfermagem.

*Enfermeira, especialista em Enfermagem Médico-Cirurgica. Mestre em Enfermagem pelo Programa de Pós-Graduação em Enfermagem da FURG. **Acadêmica do $9^{\circ}$ semestre da Faculdade de Enfermagem e Obstetrícia UFPEL/RS. Monitora da Disciplina de Enfermagem na Saúde da Criança.

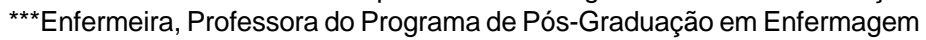
da FURG. Mestre e Doutora em Enfermagem pela UFSC/SC.

\section{INTRODUÇÃo}

Atualmente, vive-se em sociedades demasiado consumistas, em que, não raro, a insatisfação e a busca quase exclusiva de valores materiais, a intensa e incontrolável "vontade de ter cada vez mais" são bases de sustentação da vida em sociedade, que são, geralmente, responsáveis por atitudes comportamentais negativas adotadas pelas pessoas que as constituem. Com isso, surge a necessidade de dar justificativas a estas atitudes, com processos, na sua maioria, prejudiciais, tanto da ótica fisiológica quanto psicológica.

Essa busca por ter mais, muitas vezes, faz com que as pessoas procurem profissões que representam status social e conta bancária elevada, sem proporcionar, com isso, satisfação com 0 escolhido e as atividades desempenhadas, o que pode levar a conseqüências estressantes do cotidiano. Neste sentido, Leopardi (1999) diz que no século $X X$, marcado pela divisão social do trabalho, os trabalhos que não produzam riqueza são menos apreciados, bem como determinadas atividades são mais valorizadas que outras dependendo da qualidade e quantidade dos bens produzidos.

O estresse profissional, conseqüente da insatisfação profissional, pode ocasionar uma multiplicidade de fatores, como: irritabilidade, depressão ou excitação, perda de interesse, estagnação profissional e desmotivação, podendo culminar em uma baixa qualidade nos serviços prestados pelo profissional. Neste sentido, Thofhern (2002) diz que, as organizações institucionais, tornam-se um ambiente com condições negativas, 
que submetem seus clientes internos ${ }^{1}$ a uma série de tensões indevidas, acarretando danos prejudiciais ao ser humano e seu comportamento.

Segundo Heller (1999, p.8), "desde a década de 40, pesquisas sobre o comportamento humano tem revelado que diferentes necessidades estimulam as pessoas tanto no trabalho como na vida pessoal. Identificar e satisfazer esses anseios ajuda a obter o melhor de cada indivíduo". Este autor, ao comentar a teoria de Maslow sobre a hierarquia das necessidades humanas diz que, quanto mais ambiciosa e satisfeita a pessoa, maior será o seu potencial de contribuição à empresa.

Pires (1999), ao abordar o trabalho em saúde no espaço institucional, defende que, apesar da dramática realidade da saúde brasileira, a organização do trabalho nas instituições de saúde, deve ser criativo, de modo que possibilite a satisfação pessoal e a integração do potencial humano do trabalhador.

Para os trabalhadores de enfermagem, segundo Lunardi Filho (1995), o trabalho representa uma das coisas mais importantes da vida, muito embora, no cotidiano de suas atividades profissionais ele manifesta sentimentos de sofrimento e impotência frente as adversidades a sua realização.

Neste contexto, este trabalho teve a finalidade investigar a satisfação profissional e empregatícia de trabalhadores de enfermagem de um Hospital geral de uma cidade do Sul do Rio Grande do Sul.

\section{ENFATIZANDO A TRAJETÓRIA}

"Tudo nesse mundo tem seu valor. Objetos, pessoas, situações podem ter valor intrínseco, extrínseco, relativo, temporário, duradouro... Qual o valor de cada item? Como expressar o valor de cada coisa?" (Moscovici, 2001)

O sujeito trabalhador, segundo Ramos (1999), pode ser encontrado no seu mundo de trabalho, porque nele estão depositadas todas as possibilidades de construção de sua história. São várias as expressões do ser humano, das mais

${ }^{1}$ Visto neste trabalho como funcionário, especificamente da equipe de enfermagem. globais e visíveis às minúsculas e focais, mas pelo trabalho encarna tudo o que há de mais humano, em suas dimensões sociais, políticas e culturais. Assim, pode-se dizer que a procura pelo sujeito ou pelo homem significa, também, a procura pelo sujeito do trabalho ou do ser humano no trabalho.

Esta autora refere que todos precisam compreender o valor dado ao trabalho nas sociedades humanas em sua diversidade de formas de vida, em sua "imersão num universo de valores" (p.107), que não são exclusivos do trabalho, nem dele se alienam. Cezar Vaz (1999, p.58) diz que "os indivíduos se expressam vivos pela significação do trabalho e se incluem no contexto pelo significado coletivo da expressão viva do trabalho".

Leopardi (1999, p. 09), referindo ao trabalho afirma que "o trabalho, como uma atividade humana, é, por natureza, relação entre sujeito e objeto, cuja conseqüência é uma mútua transformação que se torna imediatamente movimento, de complexidade crescente".

Seguindo a idéia acima descrita, em que existe, na relação de trabalho, uma complexidade crescente, identifica-se a importância de manter, no sujeito trabalhador, prazer e envolvimento no trabalho. Neste sentido, Heller (1999) considera que quanto maior o envolvimento, maior a satisfação e a auto-realização.

A satisfação dos profissionais é um dos critérios de avaliação periódica do Sistema Nacional de Saúde, cuja legislação encontra-se na Lei $n^{\circ} 48 / 90$, de 24 de Agosto, a partir da satisfação dos clientes, da qualidade dos cuidados e da eficiente utilização dos recursos numa óptica de custo/benefício.

Acredita-se que a satisfação profissional possa ser um importante indicador da qualidade dos serviços prestados numa instituição de saúde, uma vez que quando o cliente interno está satisfeito com sua profissão e seu emprego, o envolvimento e o comprometimento com a empresa será maior.

Satisfação com o trabalho pode ser definido como um conjunto de sentimentos, favoráveis ou desfavoráveis, que os empregados nutrem em relação ao seu trabalho (DAVIS e NEWSTROM, 2001). Ela é um dos resultados da avaliação que cada trabalhador faz conforme seu grau de realização profissional, baseado em seus valores, 
necessidades, preferências e expectativas profissionais.

Corrobora-se com a assertiva de que quando uma pessoa percebe ou sente que aquilo que recebe (remuneração adequada, segurança no emprego, ambiente harmonioso no trabalho, amizade, valorização e reconhecimento profissional, oportunidade de trabalhar em equipe, entre outros) está de acordo com aquilo que esperava obter, existe maior possibilidade de encontrar-se satisfeita com a profissão escolhida e com a instituição onde trabalha.

O trabalhador da saúde, segundo Leopardi (1999) vem sendo consumido pelo trabalho, seja pelo excesso de responsabilidades, seja pelas cargas ocupacionais, como, também, pelas condições inadequadas do ambiente.

Dentre os profissionais de saúde, encontrase a equipe de enfermagem que é um grupo de pessoas formado por enfermeiros, técnicos de enfermagem e auxiliares de enfermagem, interligados por um objetivo comum: o bem estar do ser humano. Visam, também, o aprimoramento da qualidade do cuidado prestado, bem como a melhoria da qualidade de vida pessoal e profissional. Este grupo possui atribuições prédefinidas pela Lei do exercício profissional $n^{0} 7.498$, de 25 de junho de 1986 que regulamenta o exercício da profissão da enfermagem que traz no Art. $2^{\circ}$, parágrafo único: " a enfermagem é exercida privativamente pelo Enfermeiro, pelo Técnico de Enfermagem, pelo Auxiliar de Enfermagem e pela Parteira, respeitados os respectivos graus de habilitação" (COREN-RS, 1997, p.13).

No decreto regulamentador do exercício profissional $n^{0} 94.406$ de 08 de junho de 1987, em seu Art. $8^{\circ}$ consta que compete ao enfermeiro, como integrante da equipe de saúde, "participar de programas de treinamento e aprimoramento de pessoal de saúde, particularmente nos programas de educação continuada". (COREN-RS, 1997, p.1920). A profissão possui um "Código de Ética dos Profissionais de Enfermagem", conforme resolução do COFEN-160, de 12 de maio de 1993, o qual define as normas e princípios, direitos e deveres, pertinentes à conduta ética do profissional enfermeiro.

Conforme COREN-RS (1997, p.28) "A enfermagem compreende um componente próprio de conhecimentos científicos e técnicos, construído e reproduzido por um conjunto de práticas sociais, éticas e políticas que se processa pelo ensino, pesquisa e assistência. Realiza-se na prestação de serviços ao ser humano, no seu contexto e circunstâncias de vida".

Os sentimentos de satisfação (prazer) e sofrimento no trabalho da enfermagem foi foco do estudo realizado por Lunardi Filho (1995) na qual constatou que num sentido mais amplo, o trabalho atende tanto as necessidades de ordem econômica quanto emocional, assim, "não é apenas um meio de obter e garantir o sustento, mas uma forma de prazer, de auto-satisfação, de auto-valorização e realização enquanto pessoa" (p.215-216).

\section{TRILHA METODOLÓGICA}

Utilizamos para tal uma abordagem quantitativa. Chizzotti (1991), justifica que a pesquisa quantitativa "prevê a mensuração de variáveis preestabelecidas, procurando verificar e explicar sua influência sobre outras variáveis, mediante a análise da freqüência de incidências e de correlações estatísticas".

Este estudo foi realizado com profissionais de enfermagem de um Hospital Geral do Sul do Estado do Rio Grande do Sul. A amostra constou de 50 trabalhadores, escolhida, aleatoriamente, entre os profissionais de enfermagem, abrangendo os três turnos de trabalho (manhã, tarde e noite), com as três funções exercidas pela equipe de enfermagem (enfermeiro, auxiliar e técnico de enfermagem). Por meio de um encontro individual, foi explicado o objetivo do trabalho, garantido 0 anonimato e direito de desistência em qualquer etapa do estudo e permissão para publicação do mesmo. Após o Consentimento Livre e Esclarecido de todos os sujeitos escolhidos, um questionário, previamente testado, com questões sobre o tema foi aplicado, durante o turno de trabalho. O pesquisador permaneceu junto ao entrevistado durante o tempo em que o mesmo respondeu o instrumento, garantindo que todos os questionários entregues aos entrevistados fossem devolvidos, 0 que permitiu agilidade e rapidez na coleta e analise dos dados.

\section{ONDE CHEGAMOS}

As variáveis pesquisadas foram: idade, sexo 
turno de trabalho, tempo de formado, tempo de atuação na profissão, satisfação com a profissão e satisfação com o emprego atual. Dos entrevistados, $83 \%$ eram do sexo feminino e $17 \%$ masculino, sendo a média de idade é de 33,4 anos, o tempo de formado é 6,2 anos e a média de tempo de trabalho das pessoas da amostra é 5,8 anos conforme representado na quadro 1.

Quadro 1: dados referentes a idade, tempo de formado e tempo de trabalho.

\begin{tabular}{|l|c|c|c|}
\multicolumn{1}{c}{} & \multicolumn{1}{c}{ Média } & Mínima & Máxima \\
\hline Idade & 33,4 & 21 & 58 \\
\hline Tempo de formado & 6,2 & 2 & 15 \\
\hline Tempo de trabalho & 5,8 & 1 & 15 \\
\hline
\end{tabular}

A função exercida pelos componentes da amostra esta representada no gráfico 1 , sendo estes: 62\% Auxiliares de Enfermagem; 22\% Enfermeiros e 16\% são Técnicos de Enfermagem. Significa dizer que a função que absorve o maior número de pessoas que trabalham no exercício da enfermagem, atualmente, na amostra estudada, é a de auxiliares de enfermagem.

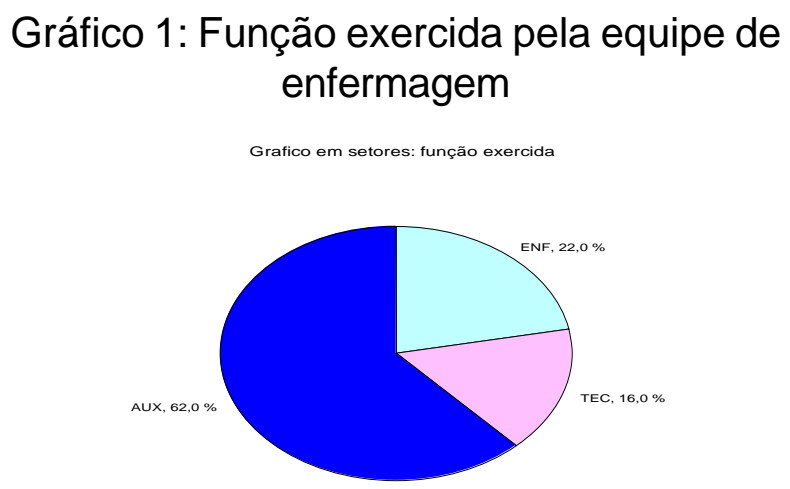

Quando analisada a variável turno de trabalho, $\mathrm{M}=$ manhã e $\mathrm{T}=$ tarde $(6$ horas trabalhadas); $\mathrm{N}=$ noite (12 horas trabalhadas), podese observa-se, no gráfico 2 que $36 \%$ da amostra trabalha no horário noturno, $32 \%$ no horário da manhã e 32\% no horário da tarde.

As demais variáveis foram agrupadas por aproximação. No item de satisfação com a profissão e satisfação com o emprego atual, foram analisados os valores atribuídos, bem como a função correspondente.

Quanto a satisfação com a profissão e a satisfação com o emprego atual, os entrevistados atribuíram um valor compreendido entre 0 (zero) e
10 (dez). Os quadros 2 e 3, respectivamente, demonstram o valor atribuído, a quantidade de profissionais, subdivididos de acordo com a função que exercem.

Quadro 2: Valor atribuído com a satisfação profissional.

\begin{tabular}{|c|c|r|}
\hline Pessoas & Função & Valor (nota) \\
\hline 1 & 1 auxiliar de enfermagem & 4,0 \\
\hline \multirow{2}{*}{2} & 3 enfermeiros & 6,0 \\
\cline { 2 - 2 } & 1 técnico de enfermagem & \\
\hline \multirow{2}{*}{13} & 1 técnico de enfermagem & \multirow{2}{*}{7,0} \\
\cline { 2 - 2 } & 1 auxiliar de enfermagem & \multirow{2}{*}{8,0} \\
\cline { 2 - 2 } & 4 enfermeiros & \\
\cline { 2 - 2 } & 1 técnico de enfermagem & \multirow{2}{*}{9,0} \\
\cline { 2 - 2 } 29 & 8 auxiliar de enfermagem & \\
\cline { 2 - 3 } & 4 enfermeiros & \\
\hline & 20 auxiliar de enfermagem & 10,0 \\
\hline 1 & 1 auxiliar de enfermagem & \\
\hline
\end{tabular}

Quadro 3: Satisfação com o emprego

\begin{tabular}{|c|c|c|}
\hline Pessoas & Função & Valor (nota) \\
\hline 2 & 1 técnico de enfermagem & \multirow{2}{*}{5,0} \\
\cline { 2 - 2 } & 1 auxiliar de enfermagem & \multirow{2}{*}{6,0} \\
\hline 6 & 4 enfermeiros & \multirow{2}{*}{7,0} \\
\cline { 2 - 2 } & 2 auxiliar de enfermagem & \multirow{2}{*}{7,0} \\
\hline \multirow{2}{*}{17} & 4 enfermeiros & \\
\cline { 2 - 2 } & 4 auxiliar de enfermagem & \\
\cline { 2 - 2 } & 2 enfermeiros & 8,0 \\
\hline & 5 técnico de enfermagem \\
\hline
\end{tabular}

A realização desse trabalho possibilitou visualizar que, de maneira geral, a categoria de técnicos e auxiliares apresentam maior satisfação profissional e empregatícia em relação ao enfermeiro. Isso nos leva a crer que é necessário um empenho maior das instituições em buscar, junto aos seus agentes colaboradores, os fatores que podem satisfazê-los no trabalho, pois acreditamos que, quanto maior a satisfação profissional, maior será sua dedicação, e, com isso, poderá prestar uma assistência de enfermagem melhor qualificada, uma vez que a satisfação reflete diretamente nas ações do trabalhador.

Esse trabalho nos instiga a investigar, por meio de um novo estudo, os motivos que influenciam na satisfação profissional e empregatícia da equipe, pois acreditamos que esse tipo de estudo, nas instituições hospitalares, pode ser utilizado como 
estratégia de medida dos fatores responsáveis pela satisfação profissional, o que nos permitiria compreender que melhorias no ambiente de trabalho poderiam ser implantadas para ampliar o grau de comprometimento do trabalhador.

ABSTRACT: The professional dissatisfaction can cause a multiplicity of negative factors interfering in the quality in the services. It is done necessary to maintain pleasure in the work, because the satisfaction and the self-realization will be larger as larger his/her involvement. This study aimed at to investigate the nursing workers' professional and employment satisfaction in a Hospital of South/RS. it was Characterized as quantitative, being used a closed questionnaire, with 50 nursing professionals. It was verified that the averages, in years, of the analyzed variables they were: 33,4 (age), 6,2 (time of having formed) and 5,8 (time of work). the distribution for function was of $22 \%$, nurses, $16 \%$ technicians and $62 \%$ auxiliary. The attributed medium value as the professional satisfaction was $7,8,8,3$ and 8,5 and for the contract of employment $6,8,7,9$ and 8,3 for, nurses, technicians and auxiliary, respectively. It is ended that the technicians and assistants present larger satisfaction degree than the nurse. That work urges us to investigate the reasons influence of the professional and employment satisfaction of the nursing team.

KEY WORDS: satisfaction; work; nursing.

\section{REFERÊNCIAS}

CEZAR VAZ, M. R. Trabalho em Saúde: expressão viva da vida social. In: LEOPARDI, M. T. (org.) O Processo de Trabalho em Saúde: organização e subjetividade. Florianópolis: Programa de Pós Graduação em Enfermagem/ UFSC. Florianópolis: Papa-Livros, 1999. p. 57-70.

CHIZZOTTI, Antônio. Pesquisa em ciências humanas e sociais. 3.ed. São Paulo: Cortez, 1991. p.15.

CONSELHO REGIONAL DE ENFERMAGEM DO RIO GRANDE DO SUL. Legislação, 1997. 48p.

DAVIS, K.; NEWSTROM, J. W. Comportamento
Humano no Trabalho: Uma abordagem Organizacional. São Paulo: Pioneira Thomson Learning, 2001.

HELLER, R. Como Motivar as pessoas. São Paulo: PubliFolha, Série Sucesso Profissional, 1999.

Lei $n^{\circ}$ 48/90, de 24 de Agosto. Leis das bases da saúde. Disponível no site: www.saude.gov.br

LEOPARDI, M. T. O Processo de Trabalho em Saúde: organização e sujetividade. Florianópolis: Programa de Pós Graduação em Enfermagem/ UFSC. Florianópolis: Papa-Livros, 1999.

LUNARDI FILHO, Wilson Danilo. Prazer e sofrimento no trabalho: contribuições À organização do processo de trabalho da enfermagem. [Dissertação] Faculdade de Ciências Econômicas - UFRGS: Porto Alegre, 1995.

MOSCOVICl, F. A Organização por traz do espelho. Rio de Janeiro: José Olympio, 2001.

PIRES, D. Aestrutura objetiva do trabalho em saúde. In: LEOPARDI, M. T. (org.) O Processo de Trabalho em Saúde: organização e subjetividade. Florianópolis: Programa de Pós Graduação em Enfermagem/ UFSC. Florianópolis: Papa-Livros, 1999 p. 25-48.

RAMOS, F. R. S. Quem produz e a quem o trabalho produz? In: LEOPARDI, M. T. (org.) O Processo de Trabalho em Saúde: organização e subjetividade. Florianópolis: Programa de Pós Graduação em Enfermagem/ UFSC. Florianópolis: Papa-Livros, 1999, p. 105-120.

THOFHERN, M. B. Construção de um modelo para o trabalho em equipe na enfermagem: ênfase nas relações interpessoais. Projeto de Tese - Doutorado em Enfermagem: UFSC, 2002. 